Trauma Berufskrankh 2007 - 9[Suppl 3]: S308-S313

DOI 10.1007/s10039-007-1265-9

Online publiziert: 22. August 2007

(c) Springer Medizin Verlag 2007

H.-H. Homann · D. Tilkorn · J. Hauser · M. Lehnhardt Universitätsklinik für Plastische Chirurgie, Schwerbrandverletzte, Handchirurgiezentrum, BG-Universitätsklinik Bergmannsheil, Ruhr-Universität, Bochum

\title{
Behandlungsoptionen bei zusätzlichem Weichteilschaden
}

Die anatomischen und funktionellen

Weichteildefekte im Fußbereich sind ein häufiges Verletzungsmuster im Rahmen von Berufsunfällen. Dabei können die Weichteilverletzungen isoliert oder begleitend zu Knochen- oder Sehnenverletzungen auftreten, was die Rekonstruktion dieser Strukturen erschwert, da ein intakter Weichteilmantel Voraussetzung zur funktionellen Wiederherstellung des Stützgerüsts und der Sehnen ist.

Der Fuß weist wichtige anatomische Besonderheiten auf, die den spezifischen funktionellen Anforderungen angepasst sind. Anatomisch der Hand ähnlich, ist der Fuß weniger ein sensibles Tast- und Greiforgan als vielmehr ein statisches Wunderwerk, das den aufrechten Gang des Menschen ermöglicht. So hat er Einfluss auf den übrigen Halte- und Stützapparat und kann bei Fehlbelastungen zu zusätzlichen Beschwerden führen.
Besonderheiten verschiedener Areale des Fußes müssen bei der Rekonstruktion des Weichteilmantels berücksichtigt werden.

\section{Fußsohle}

\section{Anatomie}

Sie verfügt über eine im Körper einzigartige Haut- und Unterhautbeschaffenheit $[3,4]$. Um den mechanischen Anforderungen zu entsprechen, ist die Haut mit einem stark ausgeprägten verhornenden Plattenepithel ausgestattet. Das Stratum corneum ist an der Fußsohle dicker als an anderen Stelle des Körpers. Das Unterhautfettgewebe besteht aus so genanntem Baufett, welches durch starke Fettgewebssepten an der kräftig ausgebildeten Plantarfaszie befestigt ist. Hierdurch sind eine optimale Verteilung und Aufnahme der Druck- und Scherkräfte, die beim Stand und Gang auftreten, sichergestellt. Die ausgeprägte Sensibilität schützt vor zu hohen Belastungen. Sensibilitätsstörungen, z. B. im Rahmen einer Polyneuropathie bei chronischen Erkrankungen wie beim Diabetes mellitus, aber auch als Folge rekonstruktiver Maßnahmen, führen daher häufig zu Ulzerationen.

\section{Rekonstruktion}

\section{Anforderungen}

Aus funktioneller Sicht lassen sich an der Fußsohle verschiedene Regionen abgrenzen. Zonen mit hoher Druckbelastung finden sich v. a. im Bereich der Ferse, dem Fußballen, also der Region der Mittfelfußköpfchen, der lateralen Fußkante und der Großzehe, während die mediale Fußkante ledig-
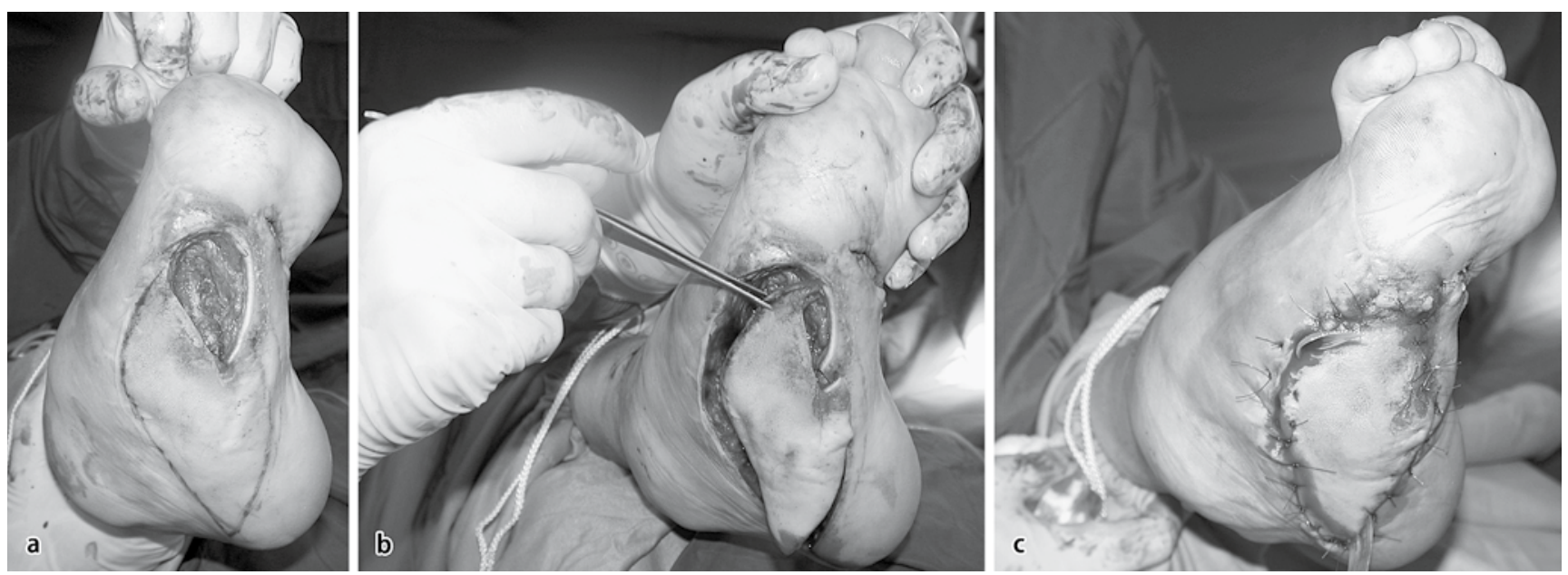

Abb. 1 \ M.-adductor-digiti-minimi(VY)-Lappen, Indikation bei distal gelegenen Defekten der lateralen Fußkante 
lich geringeren Druckanforderungen genügen muss. Aus diesen Faktoren ergibt sich:

1. Beim Débridement und der Rekonstruktion sind auch kleinste Reste originärer Sohlenweichteile erhaltenswert. Befinden sich diese neben den Lappenplastiken, können sie den Lappen entlasten und daher schonen.

2. Zur Rekonstruktion der Fußsohle sollten Verfahren angewendet werden, die eine Sensibilität gewährleisten, hohen mechanischen Ansprüchen standhalten und nur geringes Gewebeshifting verursachen, da sonst mit Ulzerationen oder instabilen Narben und Gangunsicherheit zu rechnen ist. Eine Schuhversorgung sollte bereits in die Planung der Rekonstruktion mit einfließen.

Diese Kriterien werden am idealsten von der Fußsohle selbst erfüllt.

\section{Plastiken, Verfahren und Problembereiche}

Lokale Lappenplastiken, als RandomPattern-Flaps z. B. VY-Lappen, Rotations-, Rhomboidlappen [12] usw., können erfolgreich in der Rekonstruktion kleinerer Defekte z. B. der Ballenregion eingesetzt werden ( $\bullet$ Abb. 1). Mehrzeitige Verfahren wie die Delay-Technik sorgen für eine erhöhte Sicherheit und können Lappenausmaße von $5 \mathrm{~cm} \times 6 \mathrm{~cm}$ oder $6 \mathrm{~cm} \times 8 \mathrm{~cm}$ ermöglichen.

Bei der Planung dieser Lappen ist darauf zu achten, dass Narbenverläufe möglichst außerhalb der Zonen, in denen mit einer hohen Druckbelastung zu rechnen ist, liegen. Die Hebedefekte, die in der Regel durch Spalthaut gedeckt werden können, sollten daher in die nicht so stark beanspruchte mediale Fußsohle gelegt werden.

Gefäßgestielte Nahlappen, wie v. a. der mediale Plantarlappen, sind wegen ihrer Hautqualität besonders geeignet, kleinere Defekte im Bereich der stark belasteten Ferse zu verschließen, aber auch die laterale Fußkante kann mit diesem Lappen im Mittelfußbereich eben erreicht werden. Der Hebedefekt dieses Lappens kann mittels Spalthaut verschlossen werden, als sensibler, dünner Lappen ist er für die Sohle geeignet. Der Rotationsbogen erlaubt so eine Defektdeckung im Bereich der dorsalen Ferse [13, 14].

Trauma Berufskrankh 2007 · 9[Suppl 3]: S308-S313 DOI 10.1007/s10039-007-1265-9

(c) Springer Medizin Verlag 2007

H.-H. Homann · D. Tilkorn · J. Hauser · M. Lehnhardt Behandlungsoptionen bei zusätzlichem Weichteilschaden

\section{Zusammenfassung}

Die Rekonstruktion von Weichteildefekten am Fuß stellt den Chirurgen vor diffizile Herausforderungen. Im Vordergrund der Therapie steht die Wiederherstellung der Funktion des betroffenen Fußes. Daher unterscheiden sich die Anforderungen an die Rekonstruktion von Fußrücken und -sohle. Ersterer muss eine Weichteilbedeckung zum Schutz und als Gleitlager der Strecksehnen gewährleisten, Letztere muss hohen mechanischen Belastungen standhalten, hierzu sind die meisten Transplantate nicht in der Lage. Die Teilamputation von Vorfußbereichen mit Verwen- dung des intakten Weichteilmantels zur Defektdeckung ist deshalb in das Behandlungskonzept einzubeziehen, da über die Filetlappenbildung häufig die dauerhafteste Rekonstruktion erreicht werden kann. Die Indikation zur freien Lappenplastik sollte am Fußrücken großzügig gestellt werden, dünne fasziokutane oder adipofasziale Perforatorlappen sind hierbei zu bevorzugen.

\section{Schlüsselwörter}

Fuß - Weichteildefekt · Funktion · Fußsohle . Freie Lappenplastik

\section{Treatment options in the presence of additional soft tissue damage}

\section{Abstract}

Reconstruction of soft tissue defects in the foot presents difficult challenges for the surgeon. Restoration of the function of the affected foot is the most important aim of the treatment. For this reason, the demands are different when the dorsum of the foot is to be reconstructed-this needs to provide soft tissue cover for protection and as a slipping bearing for the extendor tendons-than when the sole needs to be reconstructed. The sole of the foot has to stand up to high mechanical loads, which most transplants cannot. Partial amputation of forefoot regions with use of the intact soft tissue mantle to cover the defect can therefore be included in the treatment strategy, since the formation of fillet flaps frequently allows the most durable reconstruction. The indications for free flap transplantation on the dorsum of the foot should be interpreted liberally; thin fasciocutaneous or adipofascial perforating flaps are preferable for this purpose.

\section{Keywords}

Foot · Soft tissue defect · Function · Sole of the foot . Free flap transplantation 


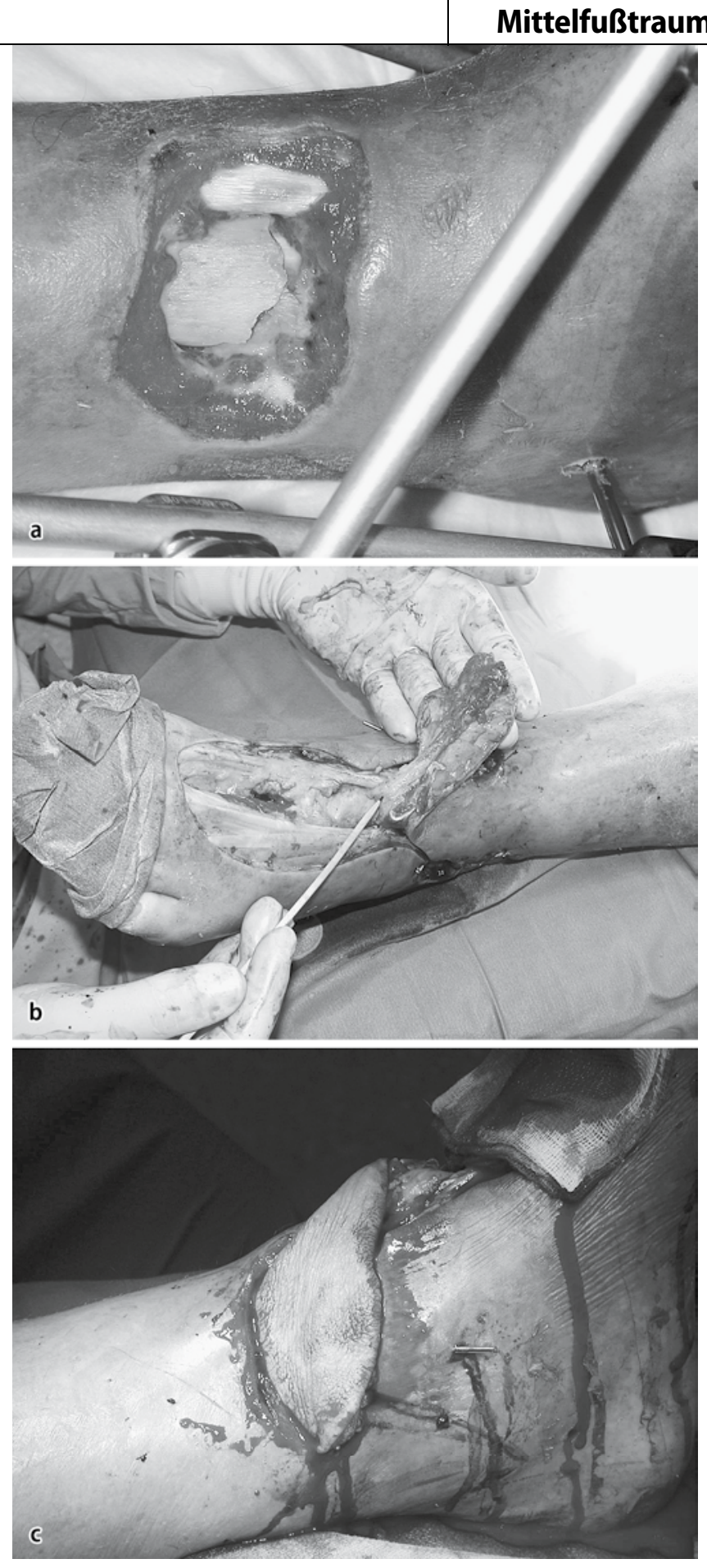

Abb. $2<$ A.-dorsalispedis-Lappen, indiziert bei Defekten der Fußränder und am distalen Drittel des Unterschenkels und der Fersenregion, im vorliegenden Fall Osteomyelitis der distalen Tibia
Der distal gestielte mediale Fußsohlenlappen reicht bis zum Fußballen. Defekte im Ballenbereich können so mit belastbarer Fußsohlenhaut verschlossen werden $[1,15]$.

Eine historisch erwähnenswerte Rekonstruktionsmöglichkeit ist der A.-dorsalis-pedis-Lappen (• Abb. 2) [16]. Als frühester beschriebener neurovaskulärer Lappen wurde er als gestielter Lappen zur Rekonstruktion des Fußrandes oder aber auch als freier Lappen verwendet. Die ho- he Morbidität und funktionellen Einbußen im Bereich des Spenderareals haben seine Indikation stark eingeschränkt, sodass er nur noch selten eingesetzt wird.

Das Hauptproblem der Defektdeckung im Sohlenbereich und hier vornehmlich in direkt belasteten Arealen, besteht im so genannten Shifting der Lappen (- Abb. 3). Darunter versteht man die Gleitfähigkeit der Gewebeschichten untereinander, an der Fußsohle ist sie durch die ausgeprägten Septen, die miteinander vernetzt sind, minimiert. Jeder andere Gewebebereich des Körpers weist ein höheres Shifting als die Sohlenhaut auf, dies kann einerseits zu Verletzungen der Haut führen, andererseits kann es aber auch zur Abscherung der Gewebeschichten untereinander kommen, entweder in Form von Pseudobursen oder aber direkter Ablösung. Um die Pseudobursenbildung zu minimieren, werden Lappen eingesetzt, die nur aus einem Gewebe bestehen, so z. B. reine Muskellappen, die dann mit Spalthaut gedeckt werden. Der Übergang der Spalthaut zum Muskel ist nicht gleitend, sodass ein Shifting nicht stattfindet. Die Spalthaut ist jedoch wenig widerstandsfähig gegenüber Druck und Abscherung, daraus resultieren eine leichte Verletzlichkeit und somit häufig offene Wunden im Belastungsareal. Eine Sensibilität existiert in reinen Muskellappen ebenfalls nicht, unabhängig von der Operationstechnik.

Der neurokutane A.-suralis-Lappen ist als gestielte Lappenplastik zur Versorgung des Mittelfußes bedingt geeignet. Basierend auf der den N. suralis begleitenden gleichnamigen Arterie wird er als fasziokutaner Lappen im distalen Muskelabschnitt des M. gastrocnemius, in Unterschenkelmittel, gehoben. Nach der Lappenpräparation erfolgt die retrograde Perfusion des Gefäßstiels über die am weitesten distal gelegene Anastomose zur A. fibularis. Diese Anastomose befindet sich etwa 3 Querfinger oberhalb des Malleolus lateralis und dient somit auch als Drehpunkt des Lappenstiels [5, 7]. Dieser Lappen kann z. B. für den lateralen Fußrand eingesetzt werden, $\mathrm{zu}$ beachten ist jedoch der ungünstige Hebedefekt. Vorteilhaft ist die erhaltene Sensibilität, gerade bei schlanken Patienten kann mit dem A.-suralis-Lappen eine gute Modellierung erreicht werden (• Abb. 4).

Vor allem am medialem Fußrand sowie anderen nicht belasteten Arealen kann bei entsprechenden Konditionen des Wundgrunds eine Spalthauttransplantation erfolgen, wobei jedoch die Wundschrumpfung in Kauf genommen werden muss und für spätere Korrekturen durchaus erwünscht ist (• Abb.5). 


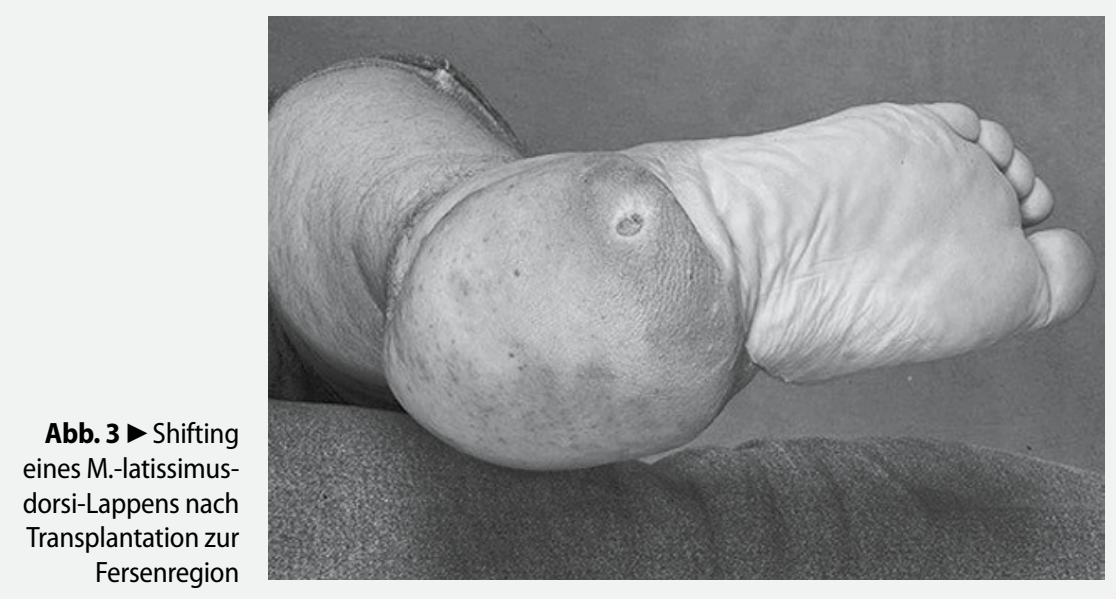

Abb. 4 A.-suralis-Lappen, indiziert bei Defekten der Ferse, der Region um die Malleolen und im Bereich des distalen Unterschenkels
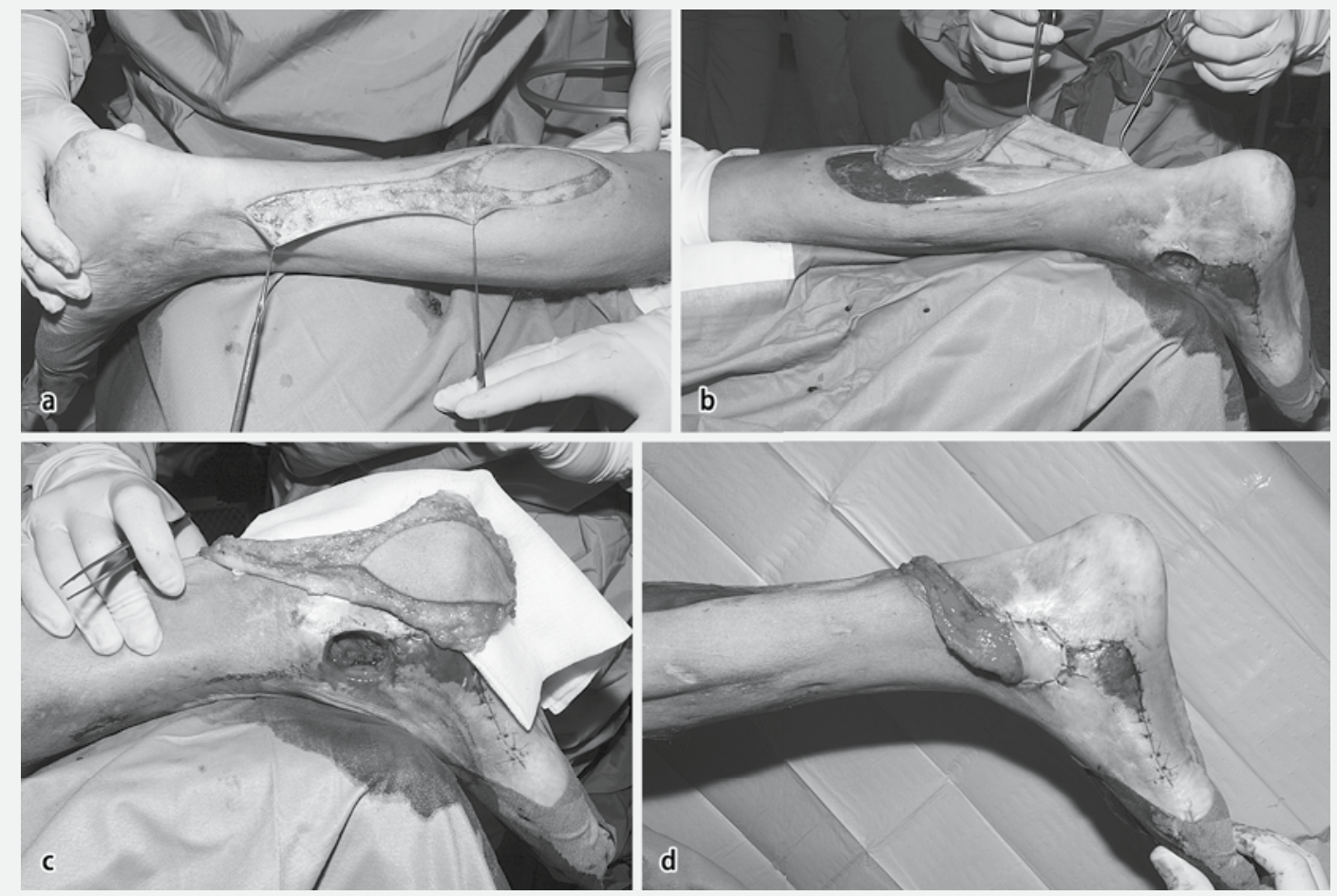

Größere Defekte besonders im Fersenbereich machen Fernlappenplastiken meist unumgänglich.

\section{Amputation}

In manchen Fällen ist es für eine funktionelle Wiederherstellung auch sinnvoll, eine Teilamputation im Sinne einer „SparePart"-Operation in Kauf zu nehmen. Ein Beispiel hierfür ist der Zehenfiletlappen (- Abb. 6). Er setzt sich aus den durch die Digitalgefäße versorgten Weichteilen nach Entfernung des Zehenskeletts zusammen. Somit steht ein sensibler gefäßgestielter Lappen mit guter Hautqualität für die Therapie von Wunden im Ballenbereich zur Verfügung [6]. Gerade bei
Patienten mit Begleiterkrankungen ist die Teilamputation des Vorfußes unter Ausnutzung der noch verbleibenden, sensiblen Weichteile zur Defektdeckung ein sicheres und schnelles Verfahren zur Rehabilitation.

Natürlich besteht eine Abneigung des Patienten und auch des behandelnden Arztes gegenüber einer Amputation, insbesondere wenn intakte Fußanteile geopfert werden. Die spätere Schuhversorgung erlaubt bei Mittelfußamputationen mit sensibler Weichteildeckung durch ortsständiges Gewebe eine nahezu uneingeschränkte Teilnahme am beruflichen und privaten Leben, da lediglich Silikoneinlagen in normalem Schuhwerk verwendet werden müssen (• Abb. 6).
Hier ist eine behutsame Aufklärung von Seiten des behandelnden Arztes notwendig, um der Teilamputation des Vorfußes den Schrecken zu nehmen.

\section{Fußrücken}

\section{Funktion und Beschaffenheit}

Der Hautmantel des Fußrückens muss ein Gleiten der Sehnen gewährleisten, während mechanische Belastungen von untergeordneter Rolle sind. Im Gegensatz zur Fußsohle ist die Haut am Fußrücken sehr dünn und weist eine große Verschieblichkeit zur Unterlage auf. 


\section{Mittelfußtraumen}
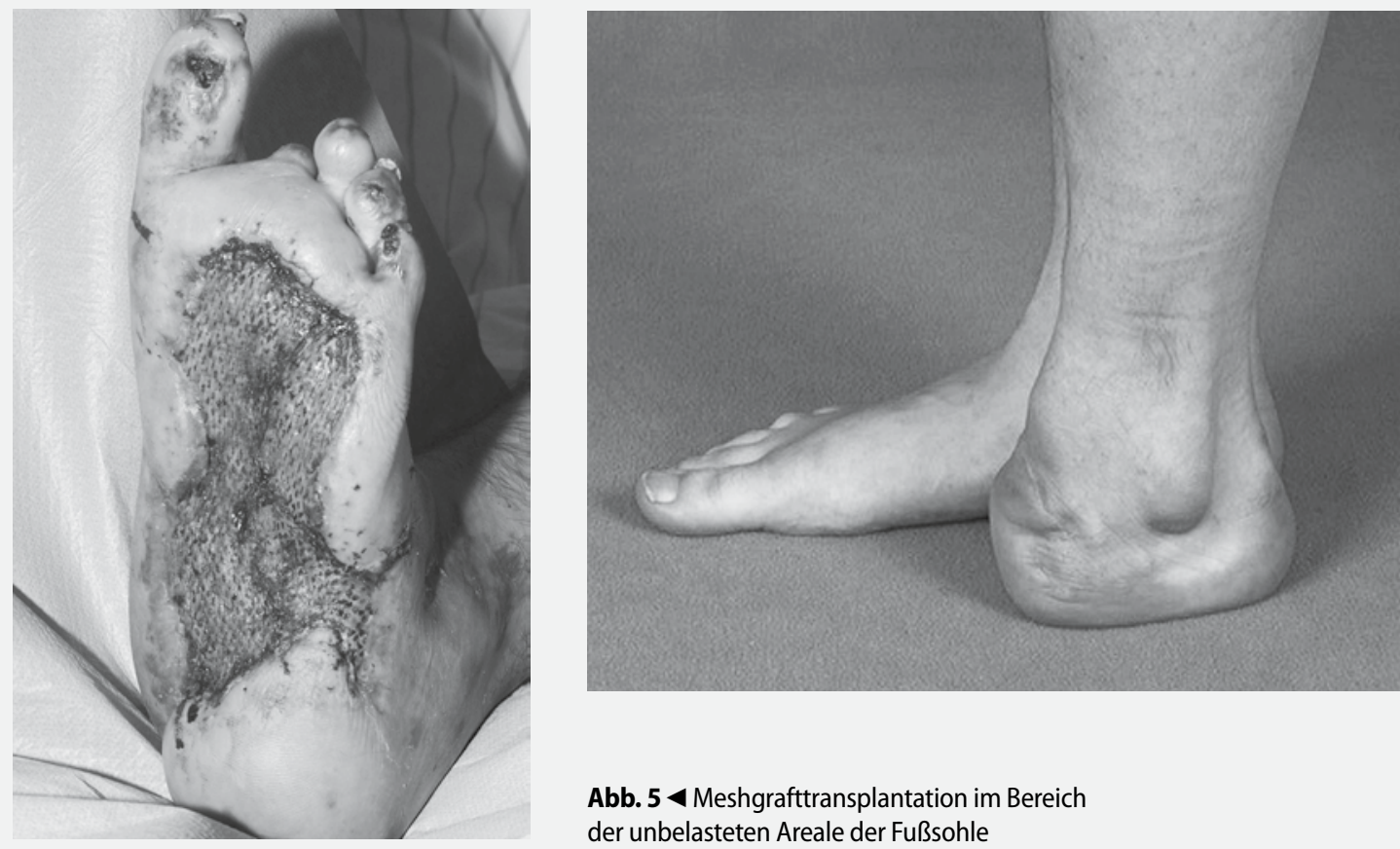

Abb. $6<$ Vorfußamputation und Defektdeckung durch Zehenfiletlappen

Abb. 54 Meshgrafttransplantation im Bereich der unbelasteten Areale der Fußsohle
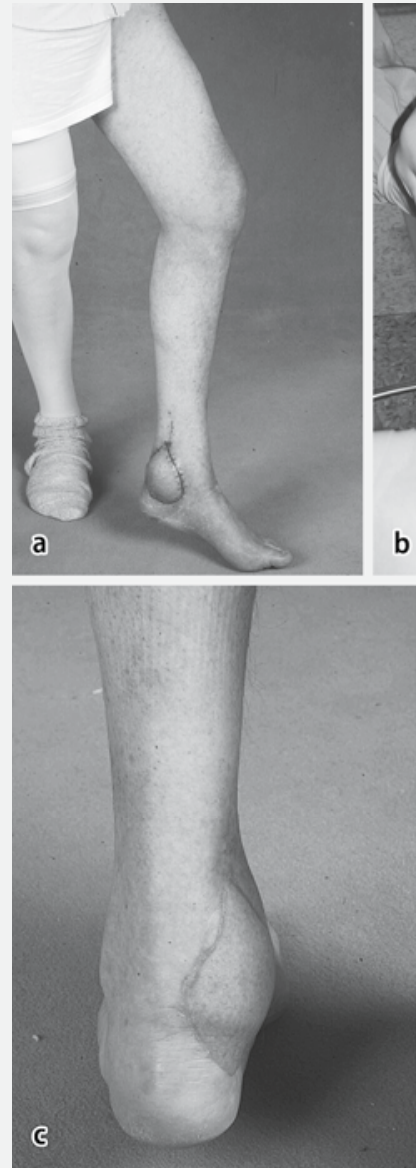
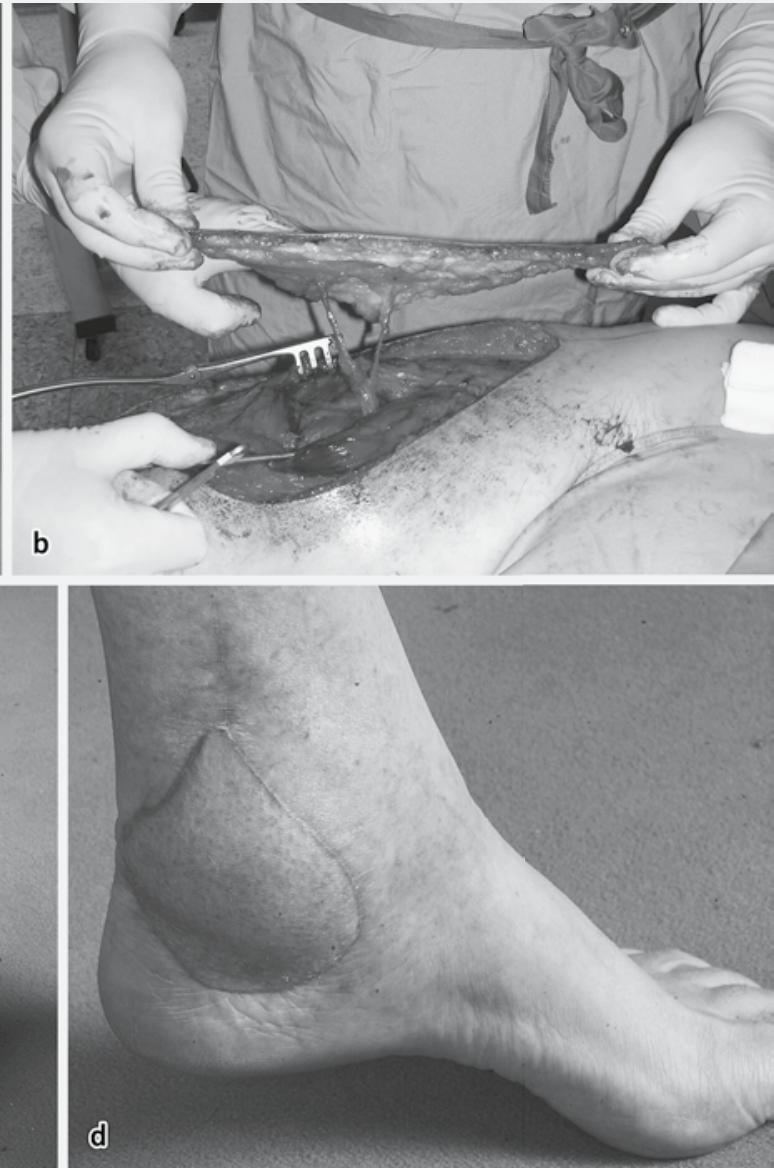

\section{Defektsanierung}

\section{Intaktes Paratenon}

Hautdefekte, bei denen das Paratenon unversehrt geblieben ist, lassen sich durch
Hautverpflanzungen erfolgreich behandeln. Liegt ein guter Wundgrund vor, sollte idealerweise eine Vollhauttransplantation durchgeführt werden, ansonsten muss eine Spalthaut als Kompromiss
Abb. $7<$ Lateraler Oberschenkellappen (, anterior lateral thigh flap", ALT) als freier, mikrochirurgisch anastomosierter Lappen

verwendet werden. Aufgrund der zu erwartenden Schrumpfungstendenz sollten Meshgrafttransplantate zurückhaltend gewählt werden. 


\section{Defektdeckung durch vaskularisiertes Gewebe}

Bei Zerstörung des Paratenons sowie bei Begleitverletzungen an Sehnen oder Knochen ist eine Defektdeckung durch vaskularisiertes Gewebe erforderlich. Posttraumatisch kann zunächst ein programmiertes, mehrfaches Débridement erforderlich sein. Eine temporäre Defektdeckung im Zeitraum dieser Wundkonditionierung mit z. B. Vakuumversiegelung sollte nur zur kurzfristigen Überbrückung angewendet werden.

Die Frührekonstruktion innerhalb der ersten 7 Tage nach dem Unfall bedeutet zum einen eine aktive Infektprophylaxe, zum anderen ist durch die fehlende Fibrosierung und Vernarbung mit einem geringeren Gewebeverlust zurechnen. $\mathrm{Zu}$ überbrückende Defektstrecken von durchtrennten Strukturen sind aufgrund der fehlenden oder geringeren Kontraktion wesentlich kürzer [10].

Die Möglichkeiten der lokalen Lappenplastiken im Bereich des Fußrückens sind jedoch sehr limitiert. Der distal gestielte als fasziokutaner Insellappen gehobene laterale Supramalleolarlappen wird durch den R. perforans der A. fibularis versorgt. Aufgrund seines großen Rotationsradius kann er im Bereich des gesamten Fußrückens verwendet werden, eine hohe Hebedefektmorbidität verbunden mit einem Gewebsüberschuss am Drehpunkt des Lappens bilden gravierende Nachteile. Als weiterer Lappen kommt der distal gestielte M.-peronaeus-brevis-Lappen in Frage, leider reicht seine Länge oft nicht zur Deckung von größeren Gewebeverlusten im Mittelfußbereich. Gleiches gilt auch für die so genannten Propellerlappen die, um $180^{\circ}$ gedreht, durch Perforansgefäße der A. tibialis posterior durchblutet werden. Mehr aus historischer Sicht interessant und heute lediglich ausgewählten Indikationen vorbehalten, soll an dieser Stelle der A.-saphena-Cross-Leg-Lappen erwähnt werden.

Häufig ist eine Versorgung mittels freien Gewebetransfers notwendig. Dieser kann die Faszie oder die Faszie einschließlich der darüber befindlichen Haut einbeziehen. Zur Auskleidung knöcherner Defekte stehen osteofasziokutane Lappen wie der Radialislappen [8], der zusammen mit einem knöchernen Anteil, und der Radi- us- oder Skapularlappen, die mit Anteilen der Spina scapulae [9] gewonnen werden können, zur Verfügung.

Eine Prämisse zur erfolgreichen Rekonstruktion des Hautweichteilmantels am Fußrücken bildet die Dicke des Lappens. Wie auch am Handrücken können hier freie Faszienlappen verwendet werden, die von der Gewebedicke her ideal sind und ein gutes Gleitlager für die Sehnen bilden. Nachteilig ist, dass sie keine epidermale Bedeckung aufweisen und somit durch eine Hauttransplantation gedeckt werden müssen. Je nach benötigter Größe sind adipooder auch fasziokutane Lappen geeignet. Bei den Perforatorlappen ist eine primäre Ausdünnung möglich, bei dem vorderen Oberschenkellappen, dem so genannten ALT-Perforatorlappen, können sehr dünne Transplantate entnommen werden (- Abb.7). Gerade bei schlanken Patienten eignen sich die Lappen hervorragend zur Rekonstruktion des Fußrückens, die Operationen können in Regionalanästhesie durchgeführt werden. Gegenüber anderen dünnen Transplantaten wie dem Radialislappen ist der Hebedefekt unserer Meinung nach günstiger, da er bis zu einer Lappenbreite von $10-12 \mathrm{~cm}$ primär verschlossen werden kann.

\section{Spenderdefekte}

Wie bei jedem rekonstruktiven Eingriff gilt es auch in der Therapie von Defekten im Fußbereich die Spenderdefekte sorgfältig in der Planung zu berücksichtigen und mit dem Patienten die Vor- und Nachteile zu diskutieren, um die ästhetischen und funktionellen Einschränkungen zu minimieren $[2,11]$.

\section{Korrespondenzadresse PD Dr. H.-H. Homann}

Universitätsklinik für Plastische Chirurgie, Schwerbrandverletzte, Handchirurgiezentrum, BG-Universitätsklinik Bergmannsheil, Ruhr-Universität, Bürkle de la Camp Platz 1, 44789 Bochum Heinz.Homann@rub.de

Interessenkonflikt. Der korrespondierende Autor gibt an, dass kein Interessenkonflikt besteht.

\section{Literatur}

1. Baker Gl, Newrton ED, Franklin JD (1990) Fasciocutaneous island flap based on the medial planta artery: clinical applications for leg, ankle and forfoot. Plast Reconstr Surg 85: 47

2. Begue T, Masquelet AC, Nordin JY (1990) Anatomical basis of the anterolateral thigh flap. Surg Radiol Anat 12: 311

3. Blechschmidt E (1932) Die Architektur des Fersenpolsters. Verhandl Anat Ges 41: 20

4. Bojsen-Moller F, Flagstadt KE (1976) Plantar aponeurosis and internal architecture of the foot. J Anat 121: 599

5. Donski PK, Fogdestam I (1983) Distally based fascio-cutaneous flap from the sural region. Scand J Plast Reconstr Surg 17: 191

6. Emmett AJJ (1976) The filete toe flap. Br J Plast Surg 29: 19

7. Fachinelli A, Masquelet A, Restrepo J et al. (1981) The vascularized sural nerv. Anatomy and surgical approach. Int J Microsurg 3: 57

8. Foucher G, Van Genechten F, Merle M et al. (1984) A compound radial artery forearm flap in hand surgery. An original modification of the Chinese forearm flap. Br J Plast Surg 37: 139

9. Gilbert A, Teot $L$ (1982) The free scapular flap. Plast Reconstr Surg 69: 601

10. Godina M (1986) Early microsurgical reconstruc tion of complex trauma of the extremities. Plast Reconstr Surg 78: 285

11. Koshima I, Fukuda H, Utunomiya R et al. (1989) The anterolateral thigh flap: variations in its vascular pedicle. Br J Plast Surg 42: 260

12. Lister G, Gibson F (1972) Closure of a rhomboid skin defects: the flaps of Limberg and Dufourmentel. Br J Plast Surg 25: 300

13. Romana MC, Masquelet AC (1989) Vascularisation of the inner border of the foot. Surgical applications. Surg Radiol Anat 2: 177

14. Shanahan RE, Gingrass R (1979) Medial plantar sensory flaps for coverage of heel defects. Plast Reconstr Surg 64: 295

15. Torii S, Namiki Y, Mori R (1987) Reverse-flow island flap: clinical report and venous drainage. Plast Reconstr Surg 79: 600

16. Zucker RM, Manktelow R (1986) The dorsalis pedisfree flap: technique of elevation, foot closure and flap application. Plast Reconstr Surg 77: 93 
Hier steht eine Anzeige.

Springer 